\title{
Influence of growth temperature on structural and optical properties of laser MBE grown epitaxial thin GaN films on a-plane sapphire
}

\author{
Chodipilli Ramesh and Prashant Tyagi \\ CSIR-National Physical Laboratory, Dr. K. S. Krishnan Road, New Delhi 110012, India and Academy of \\ Scientific and Innovative Research, CSIR-NPL Campus, Dr. K. S. Krishnan Road, New Delhi 110012, India \\ Sandeep Singh and Preetam Singh \\ CSIR-National Physical Laboratory, Dr. K. S. Krishnan Road, New Delhi 110012, India

\begin{abstract}
Govind Gupta, Kamlesh Kumar Maurya, Kuchibhotla Murali Krishna Srivatsa, Muthusamy Senthil Kumar, and Sunil Singh Kushvaha ${ }^{a}$

CSIR-National Physical Laboratory, Dr. K. S. Krishnan Road, New Delhi 110012, India and Academy of
\end{abstract} \\ Scientific and Innovative Research, CSIR-NPL Campus, Dr. K. S. Krishnan Road, New Delhi 110012, India
}

(Received 7 February 2018; accepted 26 April 2018; published 9 May 2018)

\begin{abstract}
Epitaxial thin $\mathrm{GaN}$ films $(\sim 60 \mathrm{~nm})$ have been grown on a-plane sapphire substrates at different growth temperatures $\left(500-700^{\circ} \mathrm{C}\right)$ using laser molecular beam epitaxy (LMBE). The effect of growth temperatures on the structural and optical properties of GaN layers grown on low temperature (LT) GaN buffer on prenitridated a-sapphire have been studied systematically. The in situ reflection high energy electron diffraction pattern revealed the three-dimensional epitaxial growth of $\mathrm{GaN}$ films on a-sapphire under the adopted growth conditions. The full width at half maximum (FWHM) value of x-ray rocking curves (XRCs) along GaN (0002) and (10-12) planes decreases with increasing growth temperature. The FWHM values of (0002) and (10-12) XRC for the $700{ }^{\circ} \mathrm{C}$ grown GaN film are $1.09^{\circ}$ and $1.08^{\circ}$, respectively. Atomic force microscopy characterization showed that the grain size of GaN increases from 30-60 to 70-125 nm with the increase in growth temperature as $\mathrm{GaN}$ coalescence time is shorter at high temperature. The refractive index value for the dense GaN film grown at $600^{\circ} \mathrm{C}$ is obtained to be $\sim 2.19$ at the wavelength of $632 \mathrm{~nm}$ as deduced by spectroscopic ellipsometry. Photoluminescence spectroscopy confirmed that the epitaxial GaN layers grown on a-sapphire at $600-700{ }^{\circ} \mathrm{C}$ possess near band edge emission at $\sim 3.39 \mathrm{eV}$, close to bulk GaN. The GaN growth at $700{ }^{\circ} \mathrm{C}$ without a buffer still produced films with better crystalline and optical properties, but their surface morphology and coverage were inferior to those of the films grown with LT buffer. The results show that the growth temperature strongly influences the structural and optical quality of LMBE grown epitaxial GaN thin films on a-plane sapphire, and a growth temperature of $>600^{\circ} \mathrm{C}$ is necessary to achieve good quality GaN films. Published by the AVS.

https://doi.org/10.1116/1.5025126
\end{abstract}

\section{INTRODUCTION}

Group III-nitrides (InN, GaN, and AlN) have drawn much interest in semiconductor materials after silicon due to their superior physical properties such as high breakdown field, high electron saturation velocity, radiation hardness, and good mechanical and thermal conductivities. ${ }^{1-4}$ The other electronic and electrical properties such as the direct bandgap with a large tunability and high carrier mobility lead to the fabrication of high electron mobility transistors for operation at high temperature and high frequency. ${ }^{5} \mathrm{GaN}$ and its alloys are already employed for various optoelectronic applications such as high efficient light emitting diodes (LEDs), laser diodes (LDs), monochromatic electron emission, and ultraviolet photodetector devices. ${ }^{6-8} \mathrm{GaN}$ based LED devices are mostly grown on c-plane sapphire substrates due to the nonavailability of large sized single crystalline GaN wafers. Due to the large lattice mismatch (14\%) between GaN and c-plane sapphire substrates, the a-plane sapphire can be an alternative substrate for $\mathrm{GaN}$

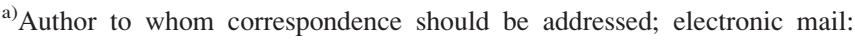
kushvahas@nplindia.org
}

growth as there is a small lattice mismatch $(<2 \%) .{ }^{9}$ The advantage of $\mathrm{GaN}$ growth on a-sapphire includes the possibility to cleave the substrate along the r-plane direction for edge-emitting LD applications. ${ }^{10}$

The epitaxial GaN layers have been grown on a-sapphire by various conventional epitaxial growth techniques such as hydride vapor phase epitaxy (HVPE), ${ }^{9,11}$ metal organic vapor phase epitaxy (MOVPE), ${ }^{11,12}$ metal organic chemical vapor deposition (MOCVD), ${ }^{13}$ and molecular beam epitaxy $(\mathrm{MBE})^{10,14}$ in the temperature range of $730-1000^{\circ} \mathrm{C}$. Even though the lattice mismatch is small for $\mathrm{GaN}$ and a-sapphire, the crystalline quality of GaN films grown on a-sapphire is comparable to that of $\mathrm{GaN}$ on c-sapphire. As per the available reports, the lowest X-ray rocking curve (XRC) full width at half maximum (FWHM) value along the GaN (0002) plane is $240 \operatorname{arc}$ sec for $12 \mu$ m thick HVPE grown $\mathrm{GaN}$ on a-sapphire. ${ }^{15}$

In recent years, laser MBE (LMBE) has been explored as a promising growth technique for low temperature (LT) growth of III-nitride layers as it offers a low temperature growth process. ${ }^{16,17}$ Highly c-axis oriented thin GaN layers grown by LMBE on c-sapphire with an XRC FWHM of 110 arc sec along the $\mathrm{GaN}$ (0002) plane have been reported. ${ }^{18}$ 
As per the available literature, the LMBE technique has been employed to grow III-nitride layers on various substrates such as $\mathrm{ZrB}_{2}$, SiC, c-sapphire, and $\mathrm{Si}^{1{ }^{18-21}}$ However, there is no report on growth of GaN on a-sapphire using LMBE yet. Here, we present the LMBE growth of the thin epitaxial GaN film $(\sim 60 \mathrm{~nm})$ on a-sapphire in a relatively low growth temperature range $\left(500-700^{\circ} \mathrm{C}\right)$ and discuss the possible growth mechanism. The effect of growth temperature on the GaN surface morphology, crystallinity, and optical properties has also been studied in detail. It is found that the LMBE has capability to grow thin c-axis oriented $\mathrm{GaN}$ films on a-sapphire at temperatures of $600-700{ }^{\circ} \mathrm{C}$ with good optical quality.

\section{EXPERIMENTAL DETAILS}

Thin epitaxial GaN films were grown on sapphire (11-20) [a-sapphire] substrates using an ultrahigh vacuum (UHV) LMBE (base pressure of $\sim 2 \times 10^{-10}$ Torr) system. ${ }^{18}$ The schematic of the $\mathrm{GaN}$ growth sequence on a-sapphire is shown in Fig. 1. The backside of the a-sapphire substrate was coated with $\sim 1 \mu \mathrm{m}$ thick molybdenum for heat absorption as an infrared heater was used for substrate heating. The substrate was thermally cleaned for $10 \mathrm{~min}$ by slowly increasing the temperature to $850^{\circ} \mathrm{C}$ in the main $\mathrm{LMBE}$ chamber under UHV conditions. The nitridation of the a-sapphire surface was performed at $700^{\circ} \mathrm{C}$ for $35 \mathrm{~min}$ by exposing active nitrogen radicals generated by an rf plasma source [1.1 sccm $\mathrm{N}_{2}$ flow, $400 \mathrm{~W} \mathrm{rf}$ power] to form few monolayer thick AlN buffer layers as shown in Fig. 1(b). The GaN film was then grown by ablating the HVPE grown polycrystalline solid GaN (99.9999\%) target using a $\mathrm{KrF}$ excimer laser at a laser repetition rate of $10 \mathrm{~Hz}$ in the presence of rf nitrogen plasma. The LT buffer GaN layers were grown on nitridated sapphire at a growth temperature of $500{ }^{\circ} \mathrm{C}$. The main $\mathrm{GaN}$ layers were grown on LT-GaN buffer layers at growth temperatures in the range of $500-700^{\circ} \mathrm{C}$ with $2 \mathrm{rpm}$ clockwise rotation of the substrate. To see the effect of the LT-GaN buffer layer, the GaN film was also grown at $700{ }^{\circ} \mathrm{C}$ without LT-GaN. The substrate cleaning, nitridation, and growth processes were monitored in situ by reflection high energy electron diffraction (RHEED) at an operating voltage of $25 \mathrm{kV}$. The thickness of LMBE grown $\mathrm{GaN}$ layers on a-sapphire is in the range of $56-60 \mathrm{~nm}$ deduced by stylus profiler and ellipsometry measurements.

The structural and optical properties of LMBE grown $\mathrm{GaN}$ films on a-sapphire were investigated using various characterization techniques such as high-resolution x-ray diffraction (HR-XRD), atomic force microscopy (AFM), spectroscopic ellipsometry (SE), and photoluminescence (PL) spectroscopy. A PANalytical HR-XRD system with a monochromatic $\mathrm{CuK}_{\alpha}$ X-ray source with the wavelength of $0.15406 \mathrm{~nm}$ was employed to characterize the crystalline quality of LMBE grown GaN films. The XRCs along the $\mathrm{GaN}$ (0002) and (10-12) diffraction planes were measured using a hybrid monochromator [multilayer graded mirror with a channel-cut two-bounce Ge (220) monochromator] in the incident beam configuration with a resolution of 12 arc sec. A scintillation detector was used to record the diffracted beam from the sample during XRC measurements. ${ }^{18}$ The surface morphologies of LMBE grown epitaxial GaN films were characterized by AFM in the tapping mode using silicon tips of curvature radii less than $10 \mathrm{~nm}$. The optical properties of GaN thin films were studied by SE, and the optical parameters were calculated in the wavelength range of 300-1000 nm for the incident angles of $55^{\circ}, 65^{\circ}$, and $75^{\circ}$. This technique is a model fitting based technique, which minimizes the difference between measured and model fitted values of ellipsometric parameters $\Psi$ (angle) and $\Delta$ (phase) as a function of wavelength or photon energy. ${ }^{22}$ The optical properties of the LMBE grown thin GaN layers were also characterized using a PL system at room temperature using a He-Cd laser $(\lambda=325 \mathrm{~nm})$ as an excitation source.

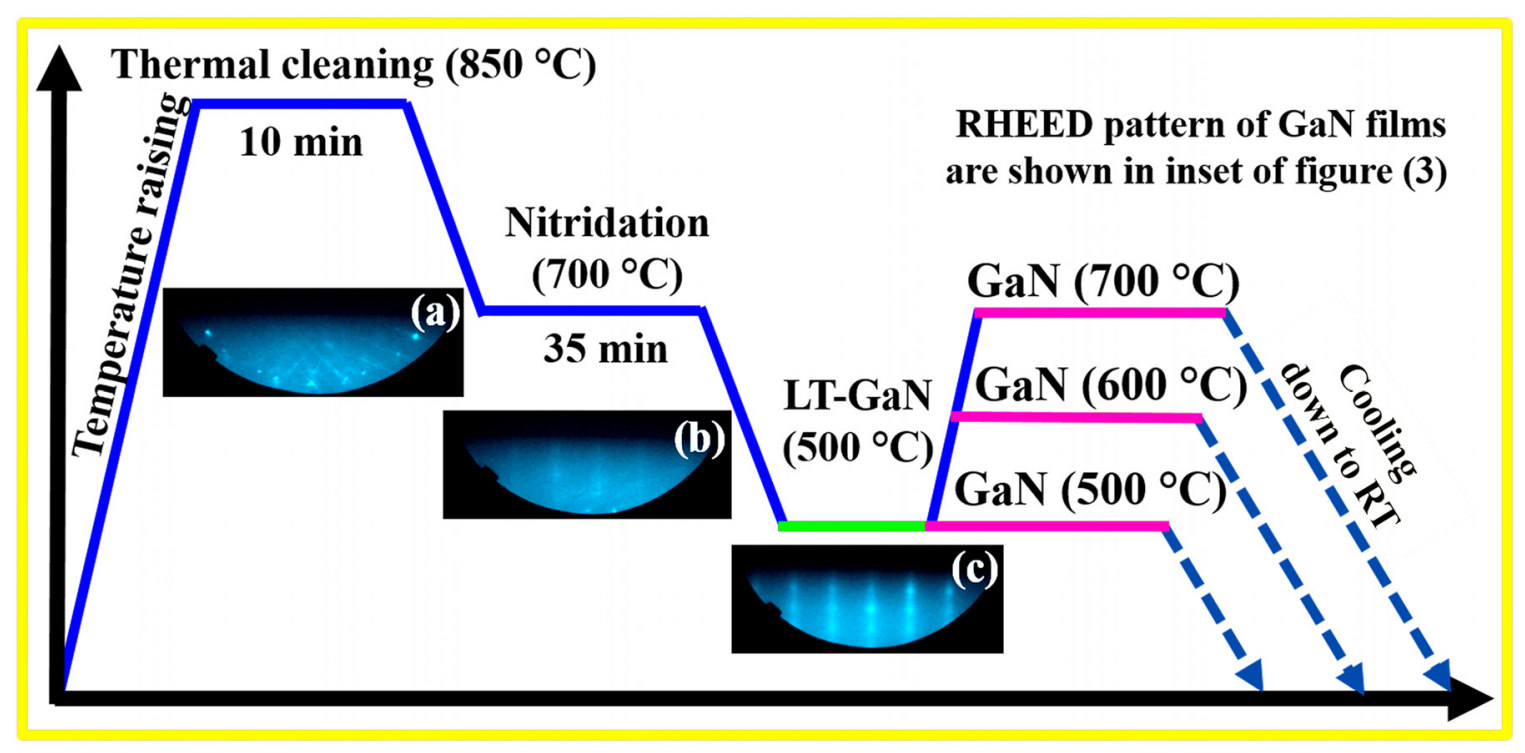

FIG. 1. (Color online) Schematic diagram of the LMBE GaN growth sequence on a-plane sapphire and its corresponding in situ RHEED patterns: (a) cleaned sapphire, (b) nitridated a-sapphire, and (c) LT-GaN grown on nitridated a-sapphire. 


\section{RESULTS AND DISCUSSION}

Figures 1(a)-1(c) show the typical RHEED patterns of the thermally cleaned a-plane sapphire substrate, nitridation, and LT-GaN buffer layer, respectively. The RHEED pattern [Fig. 1(a)] and Kikuchi lines obtained for a-sapphire revealed the clean surface achieved by thermal heating at high temperature in the UHV LMBE growth chamber. The active nitrogen radicals were exposed on thermally cleaned sapphire to form few layers of AlN on a-sapphire by replacing oxygen atoms from top of the sapphire surface. ${ }^{23}$ The sapphire substrate related RHEED short streak and Kikuchi lines became dull, and additional streak patterns corresponding to AIN appeared after nitridation of sapphire [Fig. 1(b)], indicating the conversion of the sapphire surface into AlN. The LT-GaN buffer layer growth on the prenitrided a-sapphire substrate changed the streaky pattern with the appearance of spotty features [Fig. 1(c)]. This indicates that the LT buffer GaN layer has grown three-dimensionally (3D) normal to the substrate. ${ }^{16}$ It is not surprising that the $\mathrm{GaN}$ growth occurs in the $\mathrm{N}$-rich growth condition since the polycrystalline HVPE grown stoichiometry GaN target was laser ablated in the presence of additional rf nitrogen radicals. For main GaN growth, the in situ RHEED indicated island growth with spotty features, which will be discussed in more detail in Fig. 3.

Figure 2(a) shows a typical XRD 20/ $\omega$ scan of LMBE grown GaN films on the a-sapphire substrate. The XRD pattern clearly shows that the LMBE grown GaN film on sapphire has a diffraction peak of $\mathrm{GaN}\{0001\}$ and a-sapphire \{11-20\} family of peaks, indicating the formation of coriented crystalline GaN films on the a-sapphire substrate. ${ }^{18}$ Further, the crystalline quality of $\mathrm{GaN}$ samples was examined using XRC measurements along symmetric (0002) and asymmetric (10-12) planes. The FWHM values of XRC (0002) and (10-12) planes of GaN films grown at various growth temperatures are presented in Fig. 2(b). As clearly seen, the crystalline quality of the GaN layer on a-sapphire shows a strong dependence on the growth temperature. The XRC GaN (0002) FWHM values dramatically decreased from $1.45^{\circ}$ at $500^{\circ} \mathrm{C}$ to $1.20^{\circ}-1.09^{\circ}$ at growth temperatures of $600-700^{\circ} \mathrm{C}$. The low $\left(1.03^{\circ}\right)$ XRC FWHM along GaN (0002) was obtained for the LMBE grown $\mathrm{GaN}$ at $700^{\circ} \mathrm{C}$ without LT-GaN buffer. A similar trend is also observed for the GaN (10-12) plane as shown in Fig. 2(b), and the low XRC FWHM value is obtained for the film grown at $700^{\circ} \mathrm{C}$ without the LT-GaN buffer layer. The XRC data along the $\mathrm{GaN}(10-12)$ plane for the sample grown at $500{ }^{\circ} \mathrm{C}$ could not be obtained likely due to the poor crystalline quality. The XRC measurements clearly revealed reasonably good crystalline quality of LMBE grown thin $\mathrm{GaN}$ layers on asapphire at growth temperatures of $600-700^{\circ} \mathrm{C}$. It is expected that XRC FWHM values will decrease further for thicker GaN films due to the reduction of defects in the bulk region. This result demonstrates the ability of LMBE to grow thinner $\mathrm{GaN}$ layers on a-sapphire at a low growth temperature of $700^{\circ} \mathrm{C}$. The highlight of the current study is to see the LMBE capability to grow thin epitaxial GaN films on
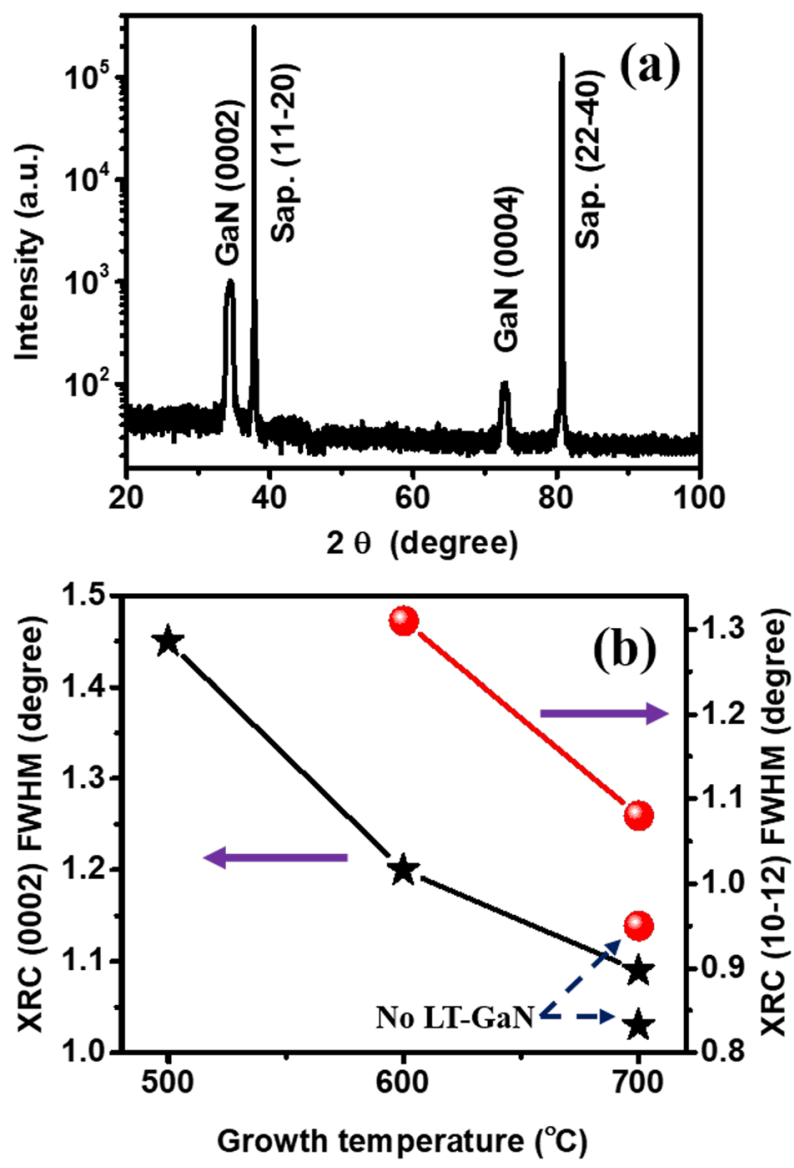

FIG. 2. (Color online) (a) Typical XRD 2 $\theta / \omega$ scan of GaN grown on the sapphire (11-20) substrate and (b) x-ray rocking curve FWHM of GaN (0002) and (10-12) planes as a function of growth temperature for LMBE grown $\mathrm{GaN}$ with LT-GaN buffer. XRC FWHM values for GaN growth at $700^{\circ} \mathrm{C}$ without the LT-GaN buffer layer are also presented.

a-sapphire at relatively low temperature for future applications in nitride based heterostructure devices.

Surface morphologies of the GaN films grown on asapphire at growth temperatures of $500-700{ }^{\circ} \mathrm{C}$ with LT-GaN buffer and without LT-GaN at $700{ }^{\circ} \mathrm{C}$ were investigated using AFM. Figure 3(a) shows the AFM image of the GaN film grown at $500^{\circ} \mathrm{C}$, and the most granular film with grain sizes ranging from 30 to $60 \mathrm{~nm}$ has been obtained. With a further increase in growth temperature to $600^{\circ} \mathrm{C}$, the grain size increased with coalesced $\mathrm{GaN}$ islands of lateral size in the range of 40 to $90 \mathrm{~nm}$ as shown in Fig. 3(b). For GaN growth at $700^{\circ} \mathrm{C}$ [Fig. 3(c)], the grain size further increased to $70-125 \mathrm{~nm}$ with few small pits on the GaN surface as observed in the AFM image. In the case of $\mathrm{GaN}$ growth at $700^{\circ} \mathrm{C}$ without LT-GaN, the sparse large $\mathrm{GaN}$ islands with a grain size of 120-180 nm were obtained [Fig. 3(d)]. The effect of $\mathrm{GaN}$ growth temperature on the crystallite size can be understood based on growth kinetics as below. The adatom or cluster diffusion on crystalline surfaces is a thermally activated process, and it also depends on the growth temperature. The surface diffusion coefficient $\mathrm{D}(\mathrm{T})$ can be expressed $\mathrm{as}^{24}$

$$
D(T)=D_{0} \exp \left[\frac{-E_{a}}{k T}\right]
$$




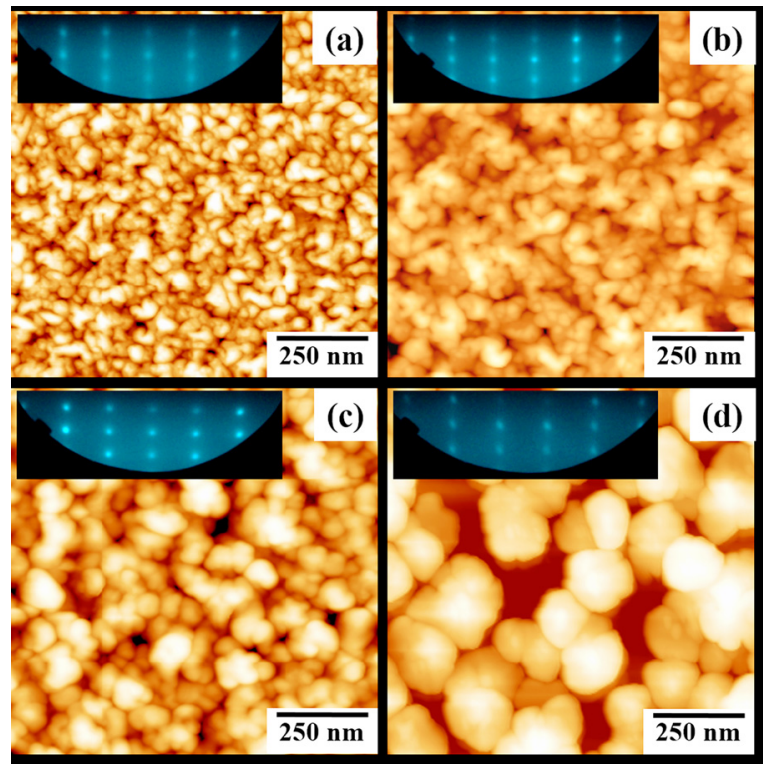

FIG. 3. (Color online) AFM images of GaN films grown on the a-plane sapphire at different growth temperatures: (a) 500, (b) 600 , and (c) $700{ }^{\circ} \mathrm{C}$ with LT-GaN buffer and (d) $700^{\circ} \mathrm{C}$ without LT-GaN buffer. Scan area: $1 \times 1 \mu \mathrm{m}$. The insets show the respective in situ RHEED patterns taken along the GaN [11-20] direction for GaN films.

where $\mathrm{D}_{0}, \mathrm{E}_{\mathrm{a}}, \mathrm{k}$, and $\mathrm{T}$ are the prefactor of the Arrhenius equation, ${ }^{24}$ activation energy, Boltzmann constant, and growth temperature, respectively. The value of $\mathrm{D}(\mathrm{T})$ increases with growth temperature, and consequently, characteristic coalescence time will be relatively short for high temperature growth. ${ }^{18,25}$ The bigger GaN grains were obtained on sapphire at higher growth temperature as the coalescence time will be short for a fixed flux in comparison to low growth temperature.

The root mean square (rms) surface roughness of thin epitaxial GaN on a-sapphire was also measured from the AFM images of $2 \times 2 \mu \mathrm{m}$ scan area. The rms surface roughness values of $\mathrm{GaN}$ layers grown at 500,600 , and $700^{\circ} \mathrm{C}$ with LT-GaN buffer are $4.13,8.24$, and $10.17 \mathrm{~nm}$, respectively. It is noticed that the size of the surface pits evolved with the increase in growth temperature, which could likely contribute to the increase in surface roughness of the grown films as measured by AFM. ${ }^{25}$ For GaN growth at $700{ }^{\circ} \mathrm{C}$ without LT$\mathrm{GaN}$, the rms surface roughness value is obtained to be $22.3 \mathrm{~nm}$. The increase in the surface roughness for high temperature-GaN growth is also supported by our in situ RHEED studies as shown in the insets of respective AFM images (Fig. 3). For example, more streaky components in the RHEED pattern were observed for the smoother GaN layer [Fig. 3(a)], whereas the rough surface possesses the spotty RHEED pattern [Fig. 3(d)]. From these observations, it is clear that the LT-GaN buffer layer promotes the growth of smooth GaN surfaces with enhanced surface coverage.

Figures 4(a) and 4(b) present the typical SE data for the ellipsometric parameters ( $\Psi$ and $\Delta$ ) of the LMBE grown $\mathrm{GaN}$ film on a-sapphire for the incident angles of $55^{\circ}$ and $75^{\circ}$. The solid line in the figure represents the model-fit data, and it can be seen that all the features present in the experimental spectra are well fitted. The thickness, refractive index, and extinction coefficient were obtained after fitting the SE data by the theoretical model fit. ${ }^{26-28}$ The fitting parameters within the parametric dispersion model yield thickness in the range of 56 to $60 \mathrm{~nm}$ for $\mathrm{GaN}$ films grown at $500-700{ }^{\circ} \mathrm{C}$, verified by stylus measurements as well. ${ }^{29}$ The variation in the refractive index and the extinction coefficient in the wavelength range of 300-1000 nm is shown in Figs. 4(c) and 4(d) after the best-fit parameters. The refractive index (thickness) values at $632 \mathrm{~nm}$ for $\mathrm{GaN}$ films grown at 500,600, and $700{ }^{\circ} \mathrm{C}$ with LT-GaN buffer and $700^{\circ} \mathrm{C}$ without LT-GaN and $3.5 \mu \mathrm{m}$ thick MOCVD grown GaN templates are 2.13 $(58 \mathrm{~nm}), 2.19(60 \mathrm{~nm}), 2.01(56 \mathrm{~nm}), 1.73(60 \mathrm{~nm})$, and 2.38 $(3.5 \mu \mathrm{m})$. The refractive index obtained for LMBE GaN films grown with the LT-GaN buffer (2.01-2.19) is relatively low compared to that of the MOCVD grown thick GaN template $(\sim 2.38)$ but is comparable to the refractive index reported for the $55 \mathrm{~nm}$ thick GaN film ( 2.21) grown on c-sapphire by low temperature atomic layer deposition. ${ }^{27}$ The variation in the refractive index value for thin $\mathrm{GaN}$ films grown at different substrate temperatures could be related to the nature of dislocations, stacking faults, grain boundary misorientation, and porosity of the films. ${ }^{26,28}$ For example, the film grown at $600^{\circ} \mathrm{C}$ showed a good surface coverage which has a higher refractive index value (2.19), whereas the film grown without LT-GaN at $700{ }^{\circ} \mathrm{C}$ revealed the low refractive index (1.73) as its surface has sparse islands with large voids as seen in AFM images (Fig. 3).

The extinction coefficient $(\mathrm{k})$ extracted from the SE data in the wavelength range of $300-1000 \mathrm{~nm}$ is shown in Fig. 4(d). The extinction coefficient is the imaginary part of the complex index of refraction, which is also related to light absorption. It defines how strongly a substance absorbs light at a given wavelength and is directly related to the absorption coefficient as follows: $:^{30}$

$$
\mathrm{k}=\alpha \lambda / 4 \pi
$$

where $\alpha$ and $\lambda$ are the absorption coefficients and wavelength, respectively. It is clear from the graphs that all the films show absorption in the ultraviolet region, but in the visible and near infrared range, the values of $\mathrm{k}$ are very low. These results indicate the minimum optical losses in these regions and also show that the films are highly transparent in the visible and near infrared range. The increase in the ultraviolet region is related to the fundamental absorption across the optical bandgap. The value decreases suddenly after the absorption edge as seen for LMBE layers and is consistent with the MOCVD GaN template. However, for the GaN film grown at $500{ }^{\circ} \mathrm{C}$, the refractive index and extinction coefficient constant values continuously increase in the lower wavelength region, which is unusual. Similar behavior of ellipsometric data has been reported for the GaN films with poor structural properties prepared by the sputtering technique. ${ }^{28}$ The band-gap of the sputtered GaN film with a high ratio of amorphous-to-crystalline phase was found to be as high as $3.47 \mathrm{eV}$ and decreased to $3.37 \mathrm{eV}$ with the increase in the crystalline phase in the film. ${ }^{28}$ Here, the unusual behavior of the LMBE grown LT GaN film may be analyzed 

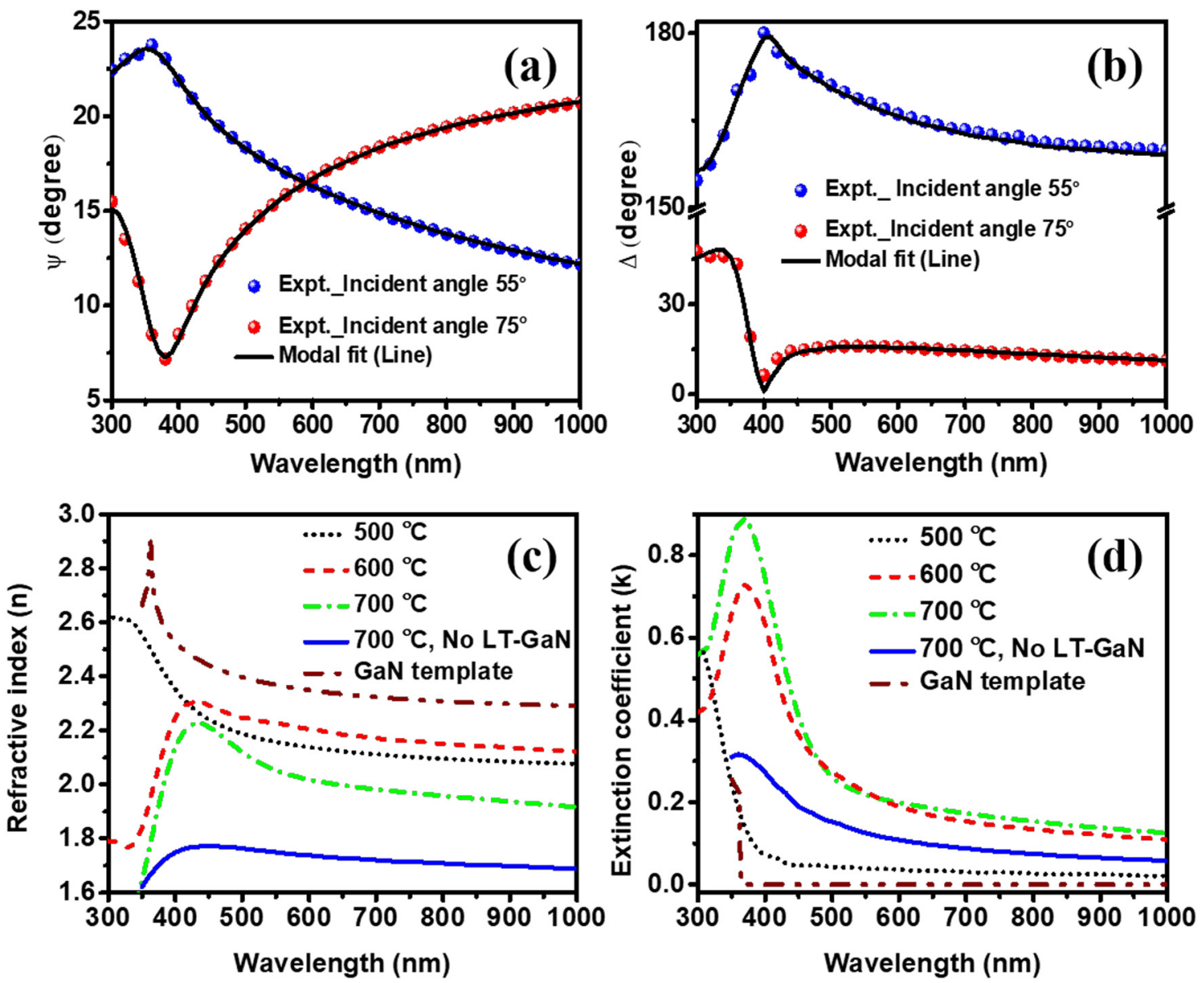

FIG. 4. (Color online) Typical experimental and model fitted ellipsometric parameters (a) $\Psi$ and (b) $\Delta$ of LMBE grown GaN thin films on a-sapphire. Solid lines indicate the theoretical model fit. Variation of (c) refractive index and (d) extinction coefficient in the wavelength range of $300-1000$ nm, extracted from the SE data for the GaN films grown at temperatures: $500-700^{\circ} \mathrm{C}$ with LT-GaN buffer and $700{ }^{\circ} \mathrm{C}$ without LT-GaN buffer. The SE data of the $3.5 \mu \mathrm{m}$ thick MOCVD grown GaN template are also included in (c) and (d) for comparison.

related to its poor crystalline quality as SE is very sensitive to the nature of the microstructure of the film.

Figure 5(a) presents the PL spectra measured for the LMBE grown epitaxial thin GaN films to study the effect of growth temperature on the light emission properties. The PL spectra for all samples were recorded with the same configuration as the PL setup at room temperature such as the incident beam, aperture size, and power of the laser excitation source. For the wurtzite bulk $\mathrm{GaN}$, the near band edge (NBE) emission PL peak is obtained at $\sim 3.4 \mathrm{eV}$, whereas the defect related PL peaks such as green and yellow luminescence (GL and YL) are observed in the range of $2.0-2.7 \mathrm{eV}^{31} \mathrm{~A}$ very broad NBE PL peak with the center at $\sim 3.05 \mathrm{eV}$ was obtained for the GaN film grown at $500^{\circ} \mathrm{C}$, which is assigned to the blue luminescence (BL) peak. With a further increase in growth temperature, i.e., $600-700^{\circ} \mathrm{C}$, the main NBE peak appears at $\sim 3.39 \mathrm{eV}$ which is nearer to bulk $\mathrm{GaN}$. The $\mathrm{GaN}$ sample grown at $700^{\circ} \mathrm{C}$ without the LT-GaN buffer also possesses the NBE peak at $3.42 \mathrm{eV}$, very close to the bulk GaN. The Lorentzian fitting of the PL NBE spectra of $\mathrm{GaN}$ grown at 600 and $700{ }^{\circ} \mathrm{C}$ also revealed the observation of the BL peak around $3.05 \mathrm{eV}$. Apart from BL peaks, we also obtained the GL peak centered at $2.54 \mathrm{eV}$ for the films grown at 500 and $600{ }^{\circ} \mathrm{C}$ with LT buffer and $700^{\circ} \mathrm{C}$ without LT buffer. The BL peaks obtained for LMBE grown $\mathrm{GaN}$ films on a-sapphire are assigned to the transition from the $\mathrm{C}_{\mathrm{Ga}}$ donor to the $\mathrm{C}_{\mathrm{N}}$ acceptor. ${ }^{31-33}$ The YL peak obtained in $\mathrm{GaN}$ films appears by transition from the shallow donor level ( $\mathrm{C}$ and $\mathrm{O}$ complexes, $\mathrm{C}_{\mathrm{N}}$ : carbon is substituted for nitrogen, and $\mathrm{O}_{\mathrm{N}}$ : oxygen is substituted for nitrogen) to deep acceptor levels (Ga vacancies). ${ }^{34,35}$ The GL defect related peak has almost a similar origin in terms of defect level transitions as of YL. ${ }^{36}$ It is reported that the GL band is related to an isolated native defect such as isolated $\mathrm{Ga}$ vacancies or isolated complexes involving Ga vacancies, whereas the $\mathrm{YL}$ is related to the same point defect bound to dislocations or possibly to structural surface defects. ${ }^{36}$

The NBE peak intensity increases with growth temperature, and it is high for the GaN film grown at $700{ }^{\circ} \mathrm{C}$. The normalized PL spectra of LMBE grown GaN films on asapphire are shown in Fig. 5(b), and it clearly shows that the FWHM of the NBE peak decreases with increasing growth temperature, e.g., $320 \mathrm{meV}$ at $600^{\circ} \mathrm{C}$ to $250 \mathrm{meV}$ at $700^{\circ} \mathrm{C}$. These observations indicate that the optical quality of the GaN film grown at $700^{\circ} \mathrm{C}$ is comparable to that of thicker GaN layers. The slightly improved crystalline and optical emission properties of the GaN film grown without buffer at $700{ }^{\circ} \mathrm{C}$ can be understood on the basis of singly grown larger 


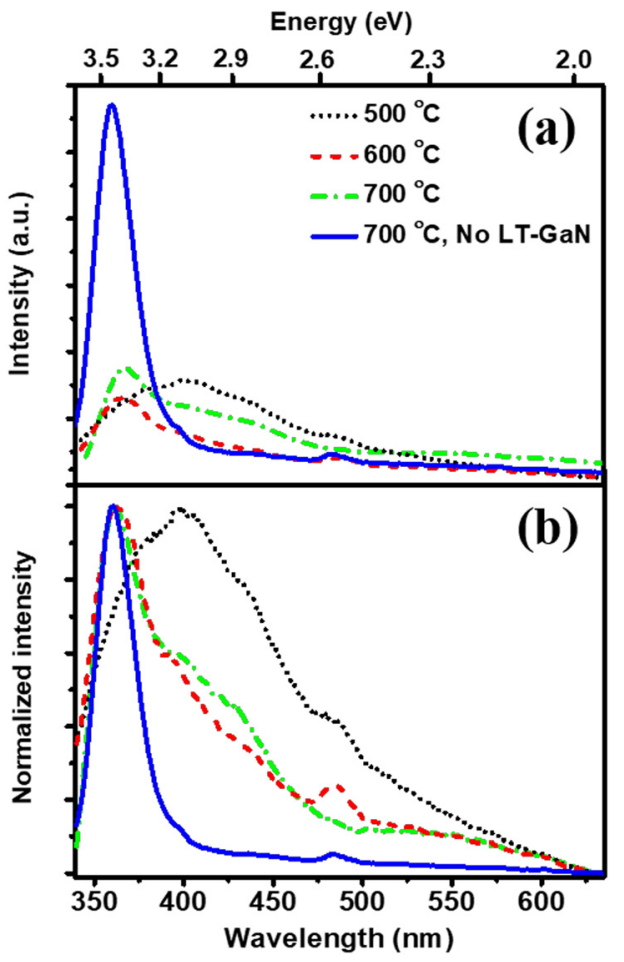

FIG. 5. (Color online) (a) As-recorded and (b) normalized room temperature PL spectra of LMBE grown GaN films on a-plane sapphire at different growth temperatures: $500-700^{\circ} \mathrm{C}$ with LT-GaN buffer and $700^{\circ} \mathrm{C}$ without LT-GaN buffer.

GaN islands that contain less structural defects. But LT-GaN buffer is essential to achieve better surface coverage and a smooth surface for device applications.

\section{SUMMARY}

We have successfully grown thin epitaxial GaN films $(\sim 60 \mathrm{~nm})$ on a-plane sapphire substrates with a LT-grown GaN buffer using the LMBE technique at growth temperatures of $500-700^{\circ} \mathrm{C}$. The HR-XRD measurements revealed the c-axis oriented GaN film growth on a-sapphire. From $\mathrm{XRC}$ measurements, it is found that the crystalline quality improved with increasing growth temperature. The AFM studies show that the size (roughness) of $\mathrm{GaN}$ islands increases from $30-60(4.13 \mathrm{~nm})$ to $70-125 \mathrm{~nm}(10.17 \mathrm{~nm})$ when the growth temperature was raised from 500 to $700{ }^{\circ} \mathrm{C}$. Room temperature PL data revealed a strong bandto-band emission at $3.39 \mathrm{eV}$ for 600 and $700{ }^{\circ} \mathrm{C}$ grown $\mathrm{GaN}$ layers along with $\mathrm{BL}, \mathrm{GL}$, and YL deep band emissions. From the results, it is found that the growth temperature strongly influences the structural, morphological, and optical properties of the LMBE grown epitaxial thin GaN films on a-sapphire and the $\mathrm{GaN}$ films grown at $600-700{ }^{\circ} \mathrm{C}$ possess good physical properties. The GaN film grown without a buffer layer exhibited better crystalline and optical properties; however, the LT-GaN buffer is found to be critical to achieve a good surface coverage. Our study demonstrates the capability of the LMBE technique to produce good quality epitaxial GaN thin films on a-sapphire at a relatively low growth temperature.

\section{ACKNOWLEDGMENTS}

The authors are grateful to Director NPL for the kind support and encouragement. Ch.R. would like to thank CSIR-SRF for financial support.

${ }^{1}$ S. Nakamura, Science 281, 956 (1998).

${ }^{2}$ Z. Liliental-Weber and H. Sohn, J. Vac. Sci. Technol., B 13, 1578 (1995).

${ }^{3}$ M. X. Feng et al., Appl. Phys. Lett. 103, 043508 (2013).

${ }^{4}$ F. A. Ponce and D. P. Bour, Nature 386, 351 (1997).

${ }^{5}$ L. K. Li, J. Alperin, W. I. Wang, D. C. Look, and D. C. Reynolds, J. Vac. Sci. Technol., B 16, 1275 (1998).

${ }^{6}$ W. Wang, Y. Lin, W. Yang, Z. Liu, S. Zhiou, H. Qian, F. Gao, L. Wen, and G. Li, J. Mater. Chem. C 2, 4112 (2014).

${ }^{7}$ S. Nakamura, M. Senoh, S. Nagahama, N. Iwasa, T. Yamada, T. Matsushita, H. Kiyoku, and Y. Sugimoto, Jpn. J. Appl. Phys., Part 2 35, L74 (1996).

${ }^{8}$ O. Yilmazoglu and L. Considine, J. Vac. Sci. Technol., B 30, 042203 (2012).

${ }^{9}$ T. Paskova, V. Darakchieva, E. Valcheva, P. P. Paskov, B. Monemar, and M. Heuken, Phys. Status Solidi B 240, 318 (2003).

${ }^{10}$ D. Doppalapudi, E. Iliopoulos, S. N. Basu, and T. D. Moustakas, J. Appl. Phys. 85, 3582 (1999).

${ }^{11}$ E. Valcheva, T. Paskova, S. Tungasmita, P. O. A. Persson, J. Birch, E. B. Svedberg, L. Hultman, and B. Monemar, Appl. Phys. Lett. 76, 1860 (2000).

${ }^{12}$ K. Doverspike, L. B. Rowland, D. K. Gaskill, and J. A. Freitas, Jr., J. Electron. Mater. 24, 269 (1995).

${ }^{13}$ T. Someya, K. Hoshino, and Y. Arakawa, Appl. Phys. Lett. 79, 1992 (2001).

${ }^{14}$ T. C. S. Krishna et al., RSC Adv. 5, 73261 (2015).

${ }^{15}$ T. Paskova, E. B. Svedberg, A. Henry, I. G. Ivanov, R. Yakimova, and B. Monemar, Phys. Scr. 79, 67 (1999).

${ }^{16}$ P. Tyagi, C. Ramesh, S. S. Kushvaha, M. Mishra, G. Gupta, B. S. Yadav, and M. Senthil Kumar, J. Alloys Compd. 739, 122 (2018).

${ }^{17}$ J. Ohta, H. Fujioka, and M. Oshima, Appl. Phys. Lett. 83, 3060 (2003).

${ }^{18}$ S. S. Kushvaha, M. S. Kumar, K. K. Maurya, M. K. Dalai, and N. D. Sharma, AIP Adv. 3, 092109 (2013).

${ }^{19}$ Y. Kawaguchi, J. Ohta, A. Kobayashi, and H. Fujioka, Appl. Phys. Lett. 87, 221907 (2005).

${ }^{20}$ M. H. Kim, M. Oshima, H. Kinoshita, Y. Shirakura, K. Miyamura, J. Ohta, A. Kobayashi, and H. Fujioka, Appl. Phys. Lett. 89, 031916 (2006).

${ }^{21}$ W. Wang, H. Wang, W. Yang, Y. Zhu, and G. Li, Sci. Rep. 6, 24448 (2016).

${ }^{22}$ C. M. Herzinger, B. Johs, W. A. McGahan, J. A. Woollam, and W. Paulson, J. Appl. Phys. 83, 3323 (1998).

${ }^{23}$ A. Georgakilas, S. Mikroulis, V. Cimalla, M. Zervos, A. Kostopoulos, P. Komninou, T. Kehagias, and T. Karakostas, Phys. Status Solidi A 188, 567 (2001).

${ }^{24}$ P. Jensen, Rev. Mod. Phys. 71, 1695 (1999).

${ }^{25}$ R. Dixit, P. Tyagi, S. S. Kushvaha, S. Chockalingam, B. S. Yadav, N. D. Sharma, and M. S. Kumar, Opt. Mater. 66, 142 (2017).

${ }^{26}$ J. H. Lee, B. Lee, J. H. Kang, J. K. Lee, and S. W. Ryu, Thin Solid Films 525, 84 (2012).

${ }^{27}$ P. Motamedi, N. Dalili, and K. Cadien, J. Mater. Chem. C 3, 7428 (2015).

${ }^{28}$ A. Biswas, D. Bhattacharya, N. K. Sahoo, B. S. Yadav, S. S. Major, and R. S. Srinivasa, J. Appl. Phys. 103, 083541 (2008).

${ }^{29}$ P. Singh, K. M. K. Srivatsa, and S. Das, Adv. Mater. Lett. 6, 371 (2015).

${ }^{30}$ H. Chu, C. Gu, S. Li, Y. Zhang, J. Bian, and C. Jiang, Opt. Mater. Express 7, 1302 (2017).

${ }^{31}$ C. H. Seager, A. F. Wright, J. Yu, and W. Götz, J. Appl. Phys. 92, 6553 (2002).

${ }^{32}$ M. A. Reshchikov and H. Morkoc, J. Appl. Phys. 97, 061301 (2005).

${ }^{33}$ M. Julkarnain, N. Kamata, T. Fukuda, and Y. Arakawa, Opt. Mater. 60, 481 (2016).

${ }^{34}$ H. C. Yang, T. Y. Lin, and Y. F. Chen, Phys. Rev. B 62, 12593 (2000).

${ }^{35}$ M. A. Reshchikov and R. Y. Korotkov, Phys. Rev. B 64, 115205 (2001).

${ }^{36}$ M. A. Reshchikov, H. Morkoc, S. S. Park, and K. Y. Lee, Appl. Phys. Lett. 78, 3041 (2001). 